\title{
Intervención en el Hogar Geriátrico San Vicente de Paúl en Barrios Altos'
}

\section{Claudia Alejandra Sánchez Grados}

Universidad de Lima, Perú

Recibido: 24 de septiembre de 2015 / Aprobado: 19 de febrero de 2016

Lima, más allá de ser una ciudad que aspira a modernizarse con el paso de los años, no puede olvidar que alberga una importante carga histórica. Es por eso que, desde 1991, y luego de diversos esfuerzos para lograrlo, el Centro Histórico de Lima entró en la lista de patrimonios mundiales reconocidos por la Unesco. Esta acción fue lograda gracias al Patronato de Lima, fundado en 1989 por un grupo de arquitectos, urbanistas, artistas, historiadores y críticos de arte, justamente para iniciar diferentes acciones de salvación de nuestro centro histórico. Sin embargo, la pregunta que muchos se hacen es: ¿qué acciones tomamos para conservar y respetar ese título? Y por otro lado, ¿la población sabe que el Centro Histórico comprende más distritos que el famoso Cercado de Lima?

Lima, Centro Histórico, Barrios Altos, restauración, Hogar Geriátrico

San Vicente de Paúl, adulto mayor

\section{Intervention at San Vicente de Paul Residence for the Elderly in Barrios Altos}

Over all these years, Lima has been a city aspiring modernization, but the city may not forget it houses an important historic burden. Since 1991, and after several efforts, Lima's Historic Center entered the list of World Heritage Sites recognized by UNESCO. Such recognition was achieved thanks to the Board of Lima founded in 1989 by a group of architects, artists, historians, and art critics who started different actions in order to rescue of our historic center. However, the question many people are asking is: On the one hand, what are we doing to protect and respect that title? And, on the other hand, do people know that this Historic Center includes other districts besides the well-known Lima Cercado?

Lima, Historic Center, Barrios Altos, restoration, San Vicente de Paul Residence for the elderly, the elderly

1 El presente artículo es parte de la tesis que viene realizando la autora para obtener la licenciatura en Arquitectura en la Universidad de Lima. 
Zonas como el Rímac, Monserrate y Barrios Altos han sido muchas veces olvidados dentro de los planes de restauración y recuperación de sus edificios, casonas o calles; por lo que actualmente se ven diferentes intervenciones que en vez de satisfacer a la población terminan destruyendo la poca esencia histórica y arquitectónica que les queda. Este es el caso del Hogar Geriátrico San Vicente de Paúl, construido en 1992 por la Sociedad de Beneficencia de Lima Metropolitana, en Barrios Altos, como un albergue para mendigos en la época. Hoy, como su nombre lo dice, recibe a adultos mayores de recursos limitados, los cuales se encuentran en ambientes que con el transcurrir de los años han sufrido diferentes modificaciones y ampliaciones arquitectónicas, sin respetarse el diseño inicial del conjunto; por lo que se pretende intervenir en él, para evaluar daños y diagnosticar el estado en que se encuentran los edificios internos, para finalmente proyectar la ampliación de un centro de día y más plazas para la residencia. Con esto, no solo se ayuda a un sector de la población poco considerado, sino que se aporta a las acciones de conservación del Centro Histórico.

\section{ELECCIÓN Y JUSTIFICACIÓN DEL TEMA}

Entendemos la adaptabilidad como la capacidad de equilibrar las progresivas disminuciones de habilidades físicas (dificultades para desplazarse, de visión, de audición, entre otras) con los cambios y situaciones extremas.

Es común constatar que las viviendas fueron pensadas para su uso inmediato, con realidades de ese momento, como las edades de sus moradores, pero prescindiendo del paso del tiempo. Esa forma de proyectarse, a menudo, olvida la etapa de la vejez con todas sus circunstancias. Lamentablemente, a los arquitectos no se les prepara para proyectar un diseño que considere la vejez, a lo más, personas con impedimentos fisicos -por lo demás normados- y en el mejor de los casos, la aplicación de una antropometría universal. Normalmente, se contempla la llegada de hijos en cantidad indeterminada. Pero de ancianidad o incapacidad, nada. (Álvarez, 2006, p. 156).

Según el INEI, en el último informe técnico de setiembre de 2014 (INEI, 2014), "el 41,4\% de los hogares del país tienen entre sus residentes habituales, al menos una persona de 60 y más años de edad. En el área rural representan el $42,3 \%$ \%. Esta relación es igual en el ámbito de Lima Metropolitana y por eso evidenciamos que la población envejece aceleradamente en relación con los años anteriores y a comparación de otros países (por ejemplo, en países europeos, considerarse adulto mayor significa tener más de 65 años).

El Perú se encuentra en un proceso de lento envejecimiento, pero activo físicamente.

El grupo de personas de 65 y más años de edad, se incrementará sostenidamente en las próximas décadas. De poco menos de 1.5 millones de adultos mayores en el 2010, se pasará a casi 6.5 millones en el 2050; 5 millones adicionales, la mayor parte de los cuales serán mujeres. (Ministerio de la Mujer y Poblaciones Vulnerables, 2013)

Es por eso que, como el mismo informe lo afirma, la mayoría de adultos mayores están presentes en los hogares y es necesario para muchas familias buscar alternativas anexas para sus cuidados.

En el ámbito de Lima Metropolitana existen solo dos grandes residencias geriátricas 
sociales para adultos mayores, pertenecientes a la Sociedad de Beneficencia de Lima. La más grande es el Albergue Central Fundación Ignacia Rodulfo Vda. de Canevaro, ubicado en el Rímac, el cual alberga a 379 adultos mayores, en su mayoría hombres, que se encuentran en la categoría social de indigentes totales o pagantes parciales; mientras que el Hogar Geriátrico San Vicente de Paúl, el más antiguo de los dos (1922), tiene a su cargo a 131 adultos mayores, en su mayoría mujeres, cuyos residentes presentan una situación de abandono familiar y social, de extrema pobreza y con presencia de salud mental deteriorada y dependencia total o parcial de cuidados médicos. Por otro lado, si hablamos del sector privado, según el Ministerio de la Mujer, existen más de quinientas residencias para adultos mayores, de las cuales solo 160 tienen licencia de funcionamiento, con comodidades que podrían superar a las de sus viviendas actuales, como el tener un dormitorio adecuadamente equipado para albergar a un adulto mayor, o contar con ambientes de interacción social entre ellos; pero con unos precios que solo podrían adecuarse a la economía del nivel socioeconómico medio y alto.

Es aquí donde se evidencia claramente el problema social por el que estamos pasando, en donde se da una mejor calidad de vida a los mejores postores. No todas las familias tienen los recursos para poder acceder a centros de cuidado del adulto mayor, o en muchos casos existe una falta de información para los usuarios, sobre diferentes programas de atención para los mismos. Es por eso que aportar en proyectos sociales en donde el adulto mayor sea el beneficiario, permitirá estandarizar los cuidados de una sociedad en donde no se debe hacer distinción por condición social, género o edad.
Por otro lado, el intervenir en un edificio existente demostrará que sí es posible recuperar lo que para muchos parece perdido y que gracias al diagnóstico e intervención arquitectónicos hay la posibilidad de rescatar de manera adecuada las edificaciones. Para esto se han utilizado los criterios de intervención para edificios de carácter histórico, los cuales son pasos que hay que seguir para una buena evaluación y próxima intervención de un edificio de carácter o valor histórico. Los objetivos fundamentales de estos criterios han sido elaborados por la Unesco y se han desarrollado en diferentes cartas de restauración por todo el mundo. Su finalidad es uniformar los tratamientos y los protocolos de acción, evitando así malas intervenciones y daños en las obras.

\section{RELACIÓN CON EL CENTRO HISTÓRICO DE LIMA}

Un factor característico de este proyecto es la ubicación actual del Hogar Geriátrico San Vicente de Paúl. La cuadra 15 del jirón Ancash no solo pertenece a Barrios Altos, además está comprendida dentro del límite de lo que hoy llamamos el Centro Histórico de Lima, reconocido como Patrimonio Mundial de la Humanidad. Sin embargo, a pesar de que este centro data del año 1922, no se encuentra en la lista de edificios declarados como patrimonio edificado. Noticia sorprendente, ya que pertenece a uno de los edificios de tipo asistencial proyectados por el arquitecto Rafael Marquina y Bueno ${ }^{2}$, quien, para la época, fue

2 Rafael Marquina y Bueno (Lima, 1884-1964). Arquitecto por la Universidad de Cornell (1909). Primer peruano que adquirió dicho título profesional. Profesor de arquitectura en la Escuela de Bellas Artes y en la Escuela de Ingenieros. Fundador de la Sociedad de Arquitectos en 1937. Arquitecto 
una de las personas más importantes en el país. Esto ha traído como consecuencia que con el transcurrir de los años las intervenciones y ampliaciones arquitectónicas realizadas han sido hechas para satisfacer la necesidad que se requería cubrir en ese momento, como nuevos ambientes o cambio de materiales en pisos y paredes, sin tomar en consideración alguna las pautas y los diseños base.

Y es que si hablamos en términos normativos, de acuerdo con la Ley $28296^{3}$, el patrimonio inmueble "comprende de manera no limitativa, los edificios, obras de infraestructura, ambientes y conjuntos monumentales, centros históricos y demás construcciones y evidencias materiales resultantes de la vida y actividad humana urbanos y/o rurales"; por lo que cualquier bien ligado a la memoria colectiva, tradiciones o costumbres, se incluye dentro de este marco. $\mathrm{Y}$ si tales bienes se quisieran intervenir, deben pasar por evaluaciones y análisis previos que recojan la mayor cantidad de información sobre el estado en que se encuentra el inmueble, para diagnosticar y actuar de manera correcta

en la Sección Técnica de la Dirección de Obras Públicas de Fomento, y luego nombrado arquitecto de la Sociedad de Beneficencia Pública de Lima, dentro del departamento de Obras Públicas. Tenía como labor la elaboración de proyectos, tasaciones, reconstrucciones, inspecciones y metrados de todas las obras que dependían de esa institución. Dentro de la Beneficencia Pública de Lima tuvo tres periodos de obras, las cuales se dividen según sus fines: de tipo asistencial, de vivienda-comercio y casas para obreros. Termina sus labores en esa institución en 1952, bajo el cargo de arquitecto consultor (Jiménez, 2005).

3 Congreso de la República Peruana (2004). Ley General del Patrimonio Cultural de la Nación, Ley N. ${ }^{0} 28296$. Consultado el 20 de enero de 2015 en <http://www.peru.gob.pe/ docs/PLANES/94/PLAN_94_LEY\%20N\%C2\%BA\%20 28296 2008.pdf $>$ sobre las acciones finales que se tomarán en su intervención. Caso contrario por el que pasó el Hogar Geriátrico San Vicente de Paúl, ya que en todas las intervenciones y ampliaciones realizadas, nunca se ha tomado en consideración evaluar los pabellones como unidades independientes y diagnosticar su estado.

Sin embargo, volvemos a la disyuntiva de por qué este centro no ha sido catalogado como patrimonio edificado, ya que su historia ha venido evolucionando con el paso de los años. Este complejo nace como un hospicio para mendigos por encargo de la Sociedad de Beneficencia Pública de Lima, bajo el diseño y la proyección del arquitecto Rafael Marquina, quien en esa época fue uno de los arquitectos más importantes debido a sus diversas obras de gran envergadura y bienestar social, que satisficieron a la población de aquel entonces. En este caso, esta obra se convirtió en uno de sus proyectos dedicados a la ayuda de tipo asistencial, pues no solo recibía a las personas, también ayudaba a cubrir todas sus necesidades básicas. Ya en los últimos años, el cambio de denominación, de residencia a hogar geriátrico, tomó además otro rol en el edificio, ya que su carácter pasó de ser residencial del todo a tener además una parte clínica. Es en este momento cuando la institución logra evidenciar su valor como contribuyente en el bienestar y desarrollo poblacional de la ciudad, en consonancia con la normativa citada. Por otro lado, su ubicación actual no resulta cualquiera, pues si se camina una cuadra de manera ascendente por el jirón Ancash, encontraremos el cementerio Presbítero Maestro, catalogado como monumento histórico por razones evidentes.

$\mathrm{Y}$ es que no solo estos factores hacen que el Hogar Geriátrico San Vicente de Paúl se haya 
podido catalogar como patrimonio, sino que la zona en conjunto, es decir, Barrios Altos, se encuentra dentro de lo que hoy llamamos Centro Histórico de Lima y alberga diferentes edificios de la época, como simples casonas o construcciones de carácter patrimonial. El caso más relevante, de la última categoría mencionada, es el cementerio Presbítero Maestro, el cual:

[...] es considerado entre los camposantos más interesantes del mundo, [...] un lugar de visita obligada en Lima. Sus calles y avenidas, como si de una ciudad se tratase, están pobladas por tumbas y mausoleos de presidentes, literatos, poetas, médicos, militares, héroes de guerras y mujeres ilustres que marcaron la historia del Perú en los siglos XIX y XX. Junto a ellos, alrededor de 220 mil personas están enterradas aquí. [...] inaugurado el 31 de mayo de 1808, cuando siguiendo la tendencia de la época, y por una necesidad de salud pública, se dejó de enterrar a los muertos en los atrios de las iglesias y se decidió abrir un camposanto en los extramuros de la ciudad de Lima, en lo que hoy es Barrios Altos. (Sociedad de Beneficencia de Lima Metropolitana, 2012)

Entonces, esto se vuelve un problema aún no resuelto: ¿por qué diferenciar arquitectura en una zona con una denominación específica?, ¿por qué no se han considerado todos los edificios de la zona como patrimonio? Y es que según el Ministerio de Cultura, al que se le fue a pedir información del complejo, no tiene registro de solicitudes hechas, por parte de ninguna entidad, para catalogarlo dentro de la lista actual de patrimonios. Por lo tanto, en el estado actual y debido a sus modificaciones realizadas, es aún difícil saber si en algún momento este centro pueda formar parte de los inmuebles intangibles y normados por dicho ministerio. Las acciones finales no solo las toma la dirección del centro, sino que es la misma Sociedad de Beneficencia de Lima la que debe realizar las acciones respectivas, con el apoyo del departamento de Obras Públicas, que está encargado de este y otros complejos.

Por otro lado, no es solo obligación de las entidades relacionadas directamente con el Hogar Geriátrico San Vicente de Paúl el recuperarlo o intervenir en él, además otras entidades públicas y privadas pueden incluir en sus planes de desarrollo a este centro, como parte de la propuesta para la zona. Tal es el caso del Ministerio de Transportes y Comunicaciones, encargado de la construcción de la Línea 1 del Metro de Lima, que construyó una de las estaciones (estación Presbítero Maestro) al lado del Hogar Geriátrico SanVicente de Paúl sin la mínima consideración para con este. Y es que no se tomó en cuenta el hecho de tener una estación de tren con cierta concurrencia de gente, que genera no solo movimiento en la zona, sino que además provoca contaminación sonora nada favorecedora para los adultos mayores. Urbanísticamente, si bien este proyecto generó un nuevo agrupamiento residencial (condominio de departamentos), poco se hizo por generar un nuevo espacio público que relacione a todos los actores de la zona (cementerio, tiendas, casas, restaurantes, etc.). Como última acción, solamente quedó, por parte del Hogar Geriátrico San Vicente de Paúl, levantar un muro ciego que limita la relación del edificio con la zona, pero que algo de protección brinda a sus residentes.

\section{DESCRIPCIÓN DEL CENTRO}

Para involucrarnos un poco más con el complejo, vale la pena describirlo en todos 
los sentidos. La planta del conjunto se diseñó organizando los volúmenes en ejes longitudinales y cuerpos transversales, que permiten separar y jerarquizar ambientes. El eje centralprincipal divide los sectores de mujeres y de hombres; mientras que los ejes secundarios, paralelos al principal, permiten tener dos volúmenes más en el área de varones y solo uno en el de mujeres. En cuanto a los cuerpos transversales, estos se definen por tres áreas. En el primero se encuentra la capilla y un área pequeña destinada actualmente para mortuorio; en el segundo cuerpo encontramos la administración; mientras que en el tercero están los talleres para terapia y servicios. Estos volúmenes, a la vez, se han ido expandiendo y modificando a lo largo de los años, por lo que actualmente se pierden estos tres últimos ejes mencionados.

En el interior del recinto existen volúmenes alargados que permiten la formación de calles internas, $y$ a los que se puede ingresar de manera lateral-central, delantera o trasera, dependiendo del caso. El material que predomina en esta construcción es el ladrillo de estilo americano, es decir, expuesto; el cual presenta un ritmo variado dependiendo de cada volumen, enmarcado por pilastras dóricas del mismo material. Las ventanas y puertas son de madera y tienen una verticalidad muy marcada, con proporciones de dos a uno. Algunas de estas, sobre todo las ventanas, presentan rejas metálicas que se han ido añadiendo con el paso de los años. Todos los edificios son de un solo piso, que, para la época, tenían una estandarización de cinco metros de altura; sin embargo, también se construyeron volúmenes de menor altura, que permitieron articular todo el conjunto. Finalmente, se plantea el tema de la verticalidad en los espacios añadiendo cornisas de madera y linternas para el ingreso de luz. A todo esto se le adhiere estructuras de carácter ornamental, como glorietas y pajareras, con alturas similares, que permiten dar proporción a los pabellones.

Este tipo de características arquitectónicas responde a la manera de pensar de Marquina, quien luego de culminar sus estudios en Estados Unidos, viene a Perú con nuevas ideas de diseño que solucionan los problemas sociales que pasaban en la época.Tal es el caso del higienismo, una corriente que se preocupa con mayor detenimiento por la salud de los habitantes y la ciudad, por lo que era necesario mantener ciertas condiciones de salubridad en los ambientes y edificios, que permitieran la correcta ventilación e iluminación de los espacios para controlar las epidemias. Por otro lado, el diseño del complejo tenía que responder a la necesidad de albergar de buena manera a la población en áreas limitadas, como respuesta al asistencialismo estatal que se

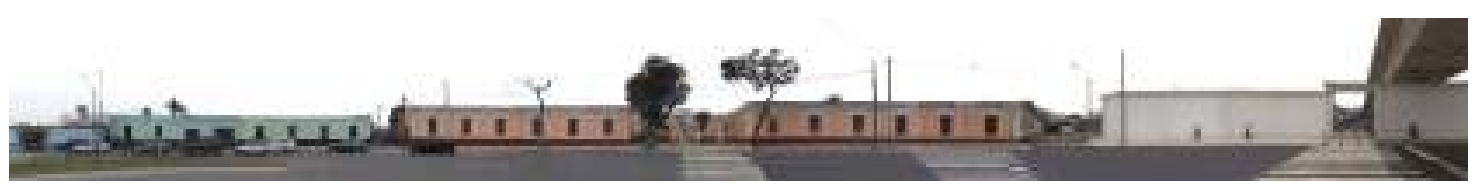

Figura 1.

Fachada

Fuente: Elaboración propia 
desató, el cual obligó al Gobierno, y en este caso a la Beneficencia Pública de Lima, a crear una arquitectura que no distinguiera comodidades entre sus usuarios.

También, como parte de las políticas del control interno de los residentes (velar por sus cuidados), respecto a la circulación se rescata que es de tipo perimetral, alrededor del eje central, para permitir el acceso libre y fluido a cualquiera de los ambientes. Esta comienza por la administración, la cual articula a todos los demás pabellones, para terminar en la sección de terapias en la parte posterior. Además, como se mencionó con anterioridad, el ingreso a los otros pabellones es puntal y definido según el eje planteado inicialmente, lo que permite pasar de un pabellón a otro sin mayor dificultad.

Este conjunto, si bien ha conservado en todos sus pabellones la esencia arquitectónica de la época, ha sufrido varias remodelaciones internas, reemplazando estructuras existentes y

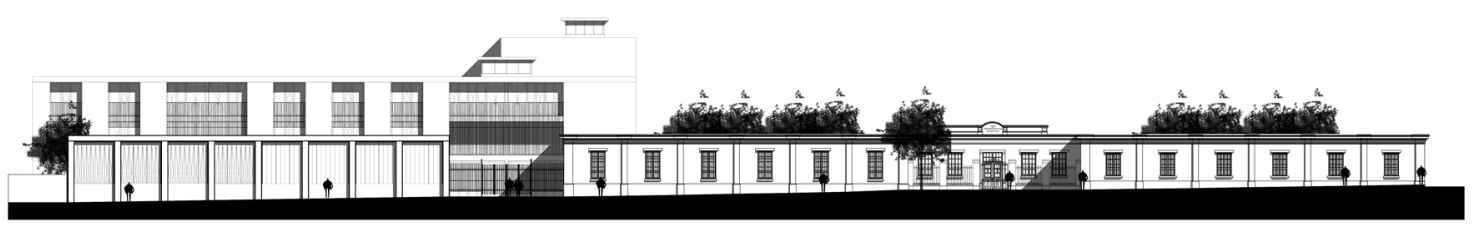

HACIA LA CALLE ANCASH

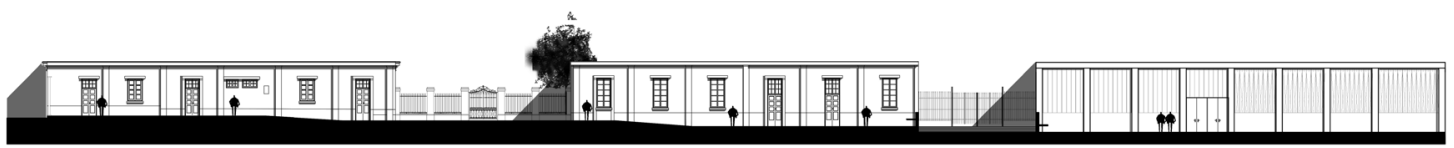

ELEVACIÓN POSTERIOR. HACIA LA NUEVA RESIDENCIA

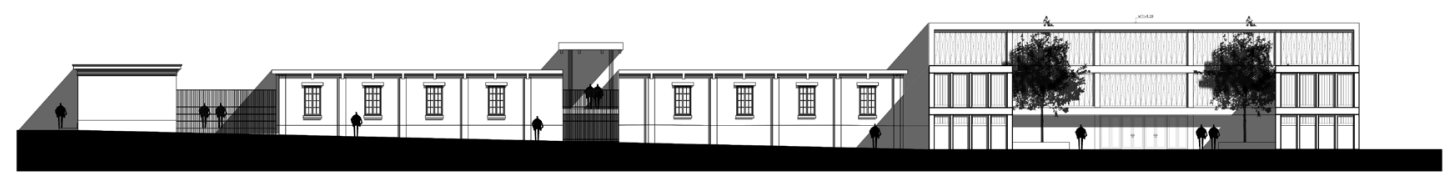

ELEVACIÓN LATERAL. CALLE RIVERA Y DÁVALOS

Figura 2.

Elevaciones del proyecto

Fuente: Elaboración propia 
añadiendo nuevos ambientes que fueron creados por un tema de necesidad y sin seguir un diseño arquitectónico correcto. Esto se debe a que todas las intervenciones realizadas, en diferentes años y periodos, han sido donaciones externas; por lo que la inversión monetaria que se les da exige un buen empleo del espacio y del dinero, pero no se tomó el diseño como un conjunto total.

Es por eso que, luego del análisis realizado, se han encontrado cuatro etapas del complejo desde su creación hasta el día de hoy. En un comienzo, eran evidentes los ejes principales y secundarios que planteó Marquina para dividir los sectores de administración y residencia de mendigos. Ya para el año 1950, se construye dentro del complejo un pabellón en donde vivían monjas cuyo servicio de caridad permitió brindar apoyo a los residentes de la época. Como tercera etapa, y ya entrando al siglo XXI, era claro que las intervenciones contemporáneas empezaron a aparecer, y es que además de realizarse una pequeña ampliación de terreno hacia el lateral izquierdo, los nuevos pabellones construidos, como lavandería, una sala multiusos y una biblioteca, ya pierden relación en cuanto altura y estética en comparación con los demás edificios en conjunto. Finalmente, ya en la actualidad y con el cambio de denominación de residencia a hogar geriátrico, el San Vicente de Paúl ha tenido que adecuar nuevas áreas de atención al residente para brindar no solo hospedaje, sino además un servicio de terapias. Esto ha
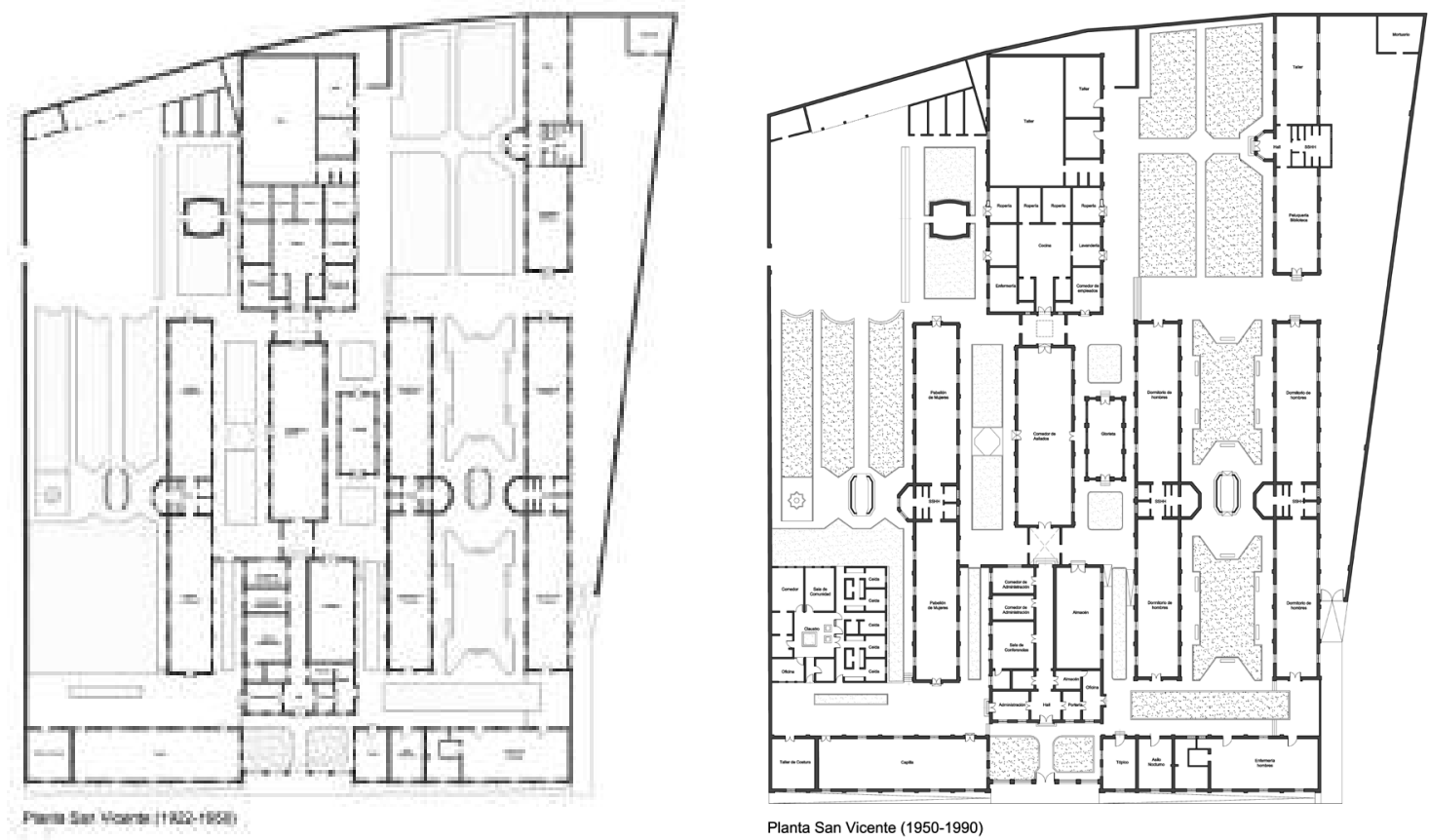


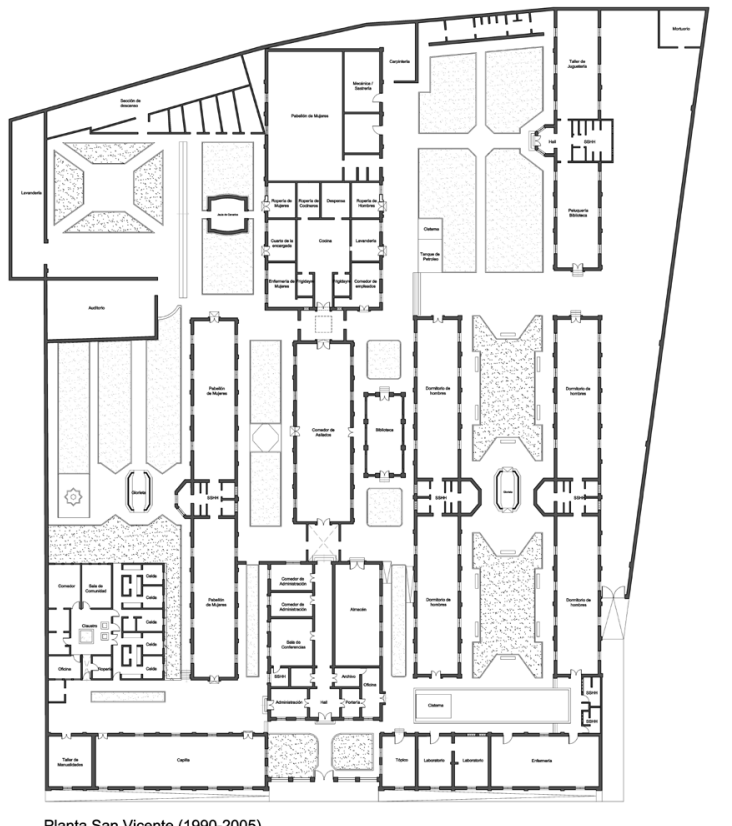

Planta San Vicente (1990-2005)

Figura 3-6.

Plantas

Fuente: elaboración propia

traído como consecuencia lo que antes se comentaba, que se ha perdido la esencia inicial del conjunto por la necesidad de responder los requerimientos que se han ido suscitando con el paso de los años.

\section{ACCIONES Y RESULTADOS FINALES}

Luego de la investigación histórica realizada sobre el complejo, fue necesario comenzar con las primeras acciones para realizar el presente proyecto de fin de carrera (tesis). Fue de gran sorpresa para mí, saber que la única información digital que tenía la Sociedad de Beneficencia de Lima Metropolitana sobre el complejo, era un

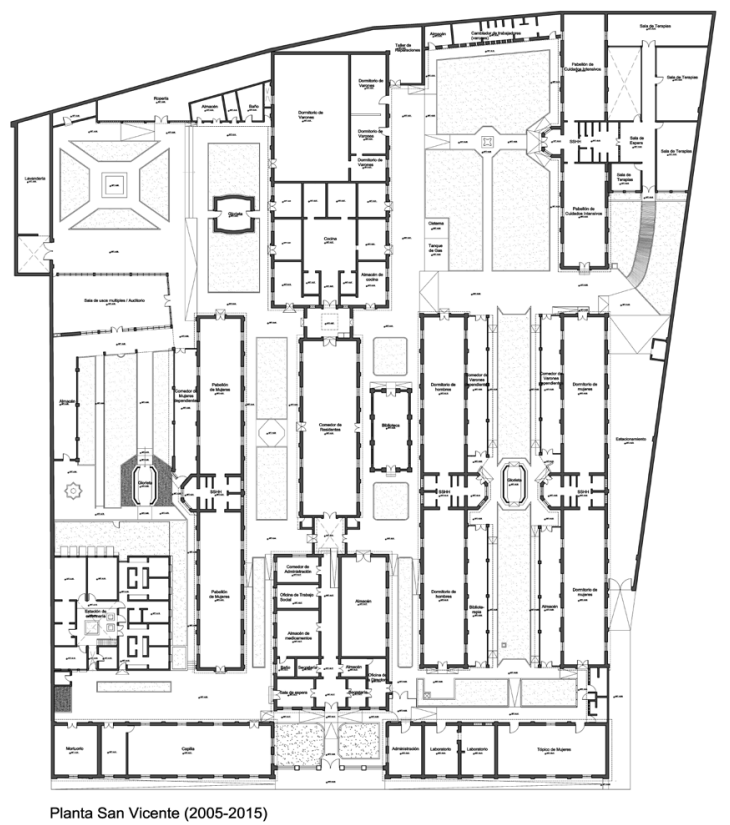

plano en planta del año 2005. Para el momento en que se comenzó este trabajo, ya habían pasado diez años de evidentes cambios, por lo que, como primera acción, se realizó la actualización del plano en planta del Hogar Geriátrico San Vicente de Paúl, para poder tenerlo como recurso principal con el cual trabajar. De manera paralela, se realizaron las mediciones correspondientes para elaborar planos de elevación de todas las fachadas de los pabellones, nuevos y antiguos, de los cuales, según la entidad encargada, jamás tuvieron registro alguno.

Culminando con este gran trabajo de medición y dibujo, se procedió a realizar el diagnóstico de daños en fachadas, carpintería, techos y pisos 
interiores de cada pabellón, lo cual permitiría evaluar el estado actual de los mismos. Para esto se elaboraron fichas de diagnóstico en las cuales se puede diferenciar el estado en el que se encuentra cada elemento arquitectónico (bueno, malo, regular, pésimo). Cabe mencionar que los diagnósticos excluyeron los ambientes interiores independientes de cada pabellón, ya que como propuesta inicial del proyecto, se pensó en el hecho de rescatar a los pabellones antiguos como envolvente, y no quedarse con muros interiores que permitieran alguna limitación en el diseño final.

Teniendo toda esta información y conociendo a la perfección todas las características del complejo, era necesario hacer la evaluación del entorno inmediato antes de pensar en el programa final del proyecto. Gracias a esto se conoció más a fondo a la población que rodea este centro, algunos edificios importantes, y se reveló además el déficit del programa arquitectónico que finalmente se adicionó al proyecto. De manera paralela, se estudiaron los factores de asoleamientos, topografia y vegetación, que han sido esenciales para establecer el diseño de fachadas y espacios públicos internos.

Con la información recaudada, se completó el programa arquitectónico que iba a tener el proyecto, el cual se divide en cuatro grandes zonas: centro de día para adultos mayores, residencia para adultos mayores, un área cultural para la población externa, y finalmente un área de interés social en la que comparten ambos actores involucrados, los adultos mayores y los vecinos de la zona. Cada uno de estos sectores tiene un ingreso controlado desde la calle, pero internamente pueden relacionarse unos con otros pasando ciertos filtros de control básicos, para asegurar la tranquilidad de los usuarios.

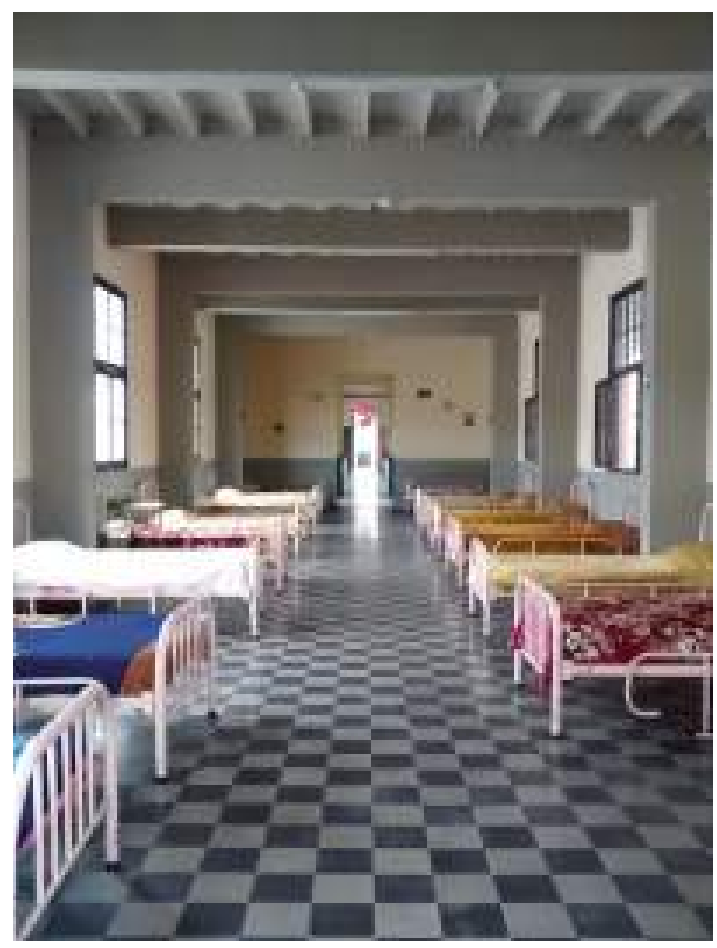

Figura 7 .

Interior del Hogar

Fuente: Fotografía de la autora

Todo lo mencionado termina relacionándose con el entorno inmediato y permite convertir el proyecto en un punto importante de anclaje e interconexión de programas.

Como se mencionó desde un principio, el Hogar Geriátrico San Vicente de Paúl no presenta carácter de patrimonio histórico, sin embargo, a pesar de eso, se decidió considerarlo como tal para poder rescatar la esencia arquitectónica de la época. Es por eso que el proyecto comienza con la limpieza total del complejo, para quedarse solo con los pabellones que Marquina diseñó inicialmente (a excepción de uno) y comenzar con el 


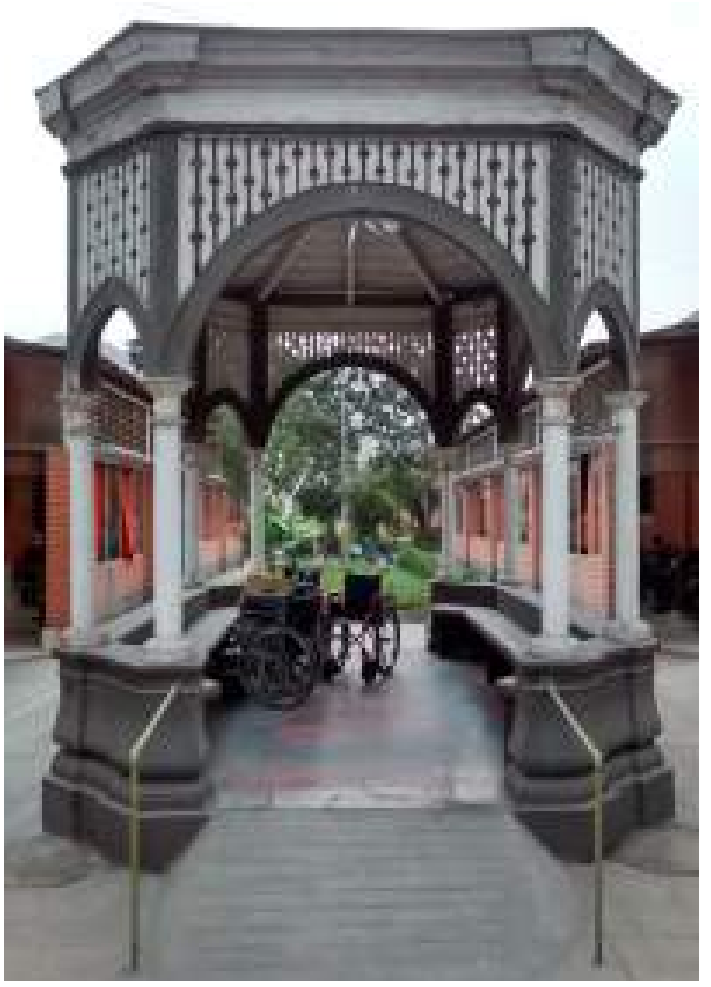

Figura 8.

Glorieta

Fuente: Fotografía de la autora

diseño de la obra nueva. Al tener menos área construida existente, se decide colocar toda la parte cultural en los pabellones antiguos y proyectar las zonas de centro de día, residencia y social, en la arquitectura nueva. Es importante mencionar que por un tema de área necesaria en el proyecto y la estrategia de relación con el entorno, la ampliación del proyecto se tuvo que realizar hacia la parte izquierda del terreno actual, y se tiene así mayor cantidad de metraje en fachada para el jirón Ancash.
Finalmente, el proyecto comienza a nacer basándose en los ejes principales existentes de la arquitectura antigua, complementando con ejes secundarios que permiten lograr cerramientos específicos y delimitación de las zonas mencionadas. Por otro lado, apoyándose en la normativa que dicta la Municipalidad de Lima sobre los reglamentos de zonificación, el proyecto termina con una altura máxima de cuatro pisos, retirados de las fachadas, ya que en estas solo se permitía como máximo un piso de doble altura o dos pisos de tamaño normal. Como un servicio adicional (ya que no lo estipula el reglamento de la zona), se proyectó un estacionamiento subterráneo que servirá exclusivamente para las personas que llegarán a recibir atención en el centro de día o que visitarán a sus familiares en la residencia.

Para la fecha actual de redacción del presente artículo, el diseño del proyecto sigue en elaboración; y con miras a una próxima sustentación oral, se espera que dicho proyecto no solo sirva como ejemplo para futuros tesistas de la Universidad de Lima, por ser uno de los primeros trabajos sustentados por una egresada de la primera promoción de la Carrera de Arquitectura, sino que además pueda servir para concientizar sobre la existencia de edificios que quizá no sean patrimonio histórico, pero que se pueden rescatar de una u otra manera, para planear nueva arquitectura. Por último, también cabe mencionar que se debe pensar en atender a todo tipo de población; y es que brindar calidad de vida significa no excluir a nadie por ninguna limitación, condición fisica o edad. Tenemos la obligación, como personas y profesionales de diferentes carreras, de atender de manera adecuada a los adultos mayores, hasta su partida. 


\section{REFERENCIAS}

Álvarez, F. (2006). El adulto mayor, su entorno y su vivienda. El buen envejecer. Gerontología, diplomado de gerontología a distancia. Santiago: Pontificia Universidad Católica de Chile.

Cisneros, L.J. (2000). Lima: el Centro Histórico, espacio de vida. El Correo de la Unesco, 63-64.

Congreso de la República Peruana. (2004). Ley General del Patrimonio Cultural de la Nación, Ley N. ${ }^{\circ}$ 28296. Recuperado de <http://www.peru.gob.pe/docs/ PLANES/ 94 / PLAN_94_LEY\%20 N\%C2\%BA\%2028296_2008.pdf>

INEI. (septiembre, 2014). Situación de la población adulta mayor. Informe técnico n. ${ }^{\circ}$ 4. Lima: autor.

Jiménez,V. (2005). Rafael Marquina, arquitecto. Lima: Fondo Editorial Universidad Nacional de Ingeniería.

Ministerio de Cultura. (2015). Patrimonio cultural. Recuperado de <http://www.cultura. gob.pe/es/patrimonio/historicoinmueble/ conceptosydefiniciones>
Ministerio de la Mujer y Poblaciones Vulnerables. (2013). Cuadernos sobre poblaciones vulnerables n. ${ }^{\circ} 5$ - PLANPAM 2013-2017. Lima: autor.

Ruiz Rodrigo, C., \& Palacio Lis, I. (1999). Higienismo, educación ambiental y previsión escolar: antecedentes. España: Universidad de Valencia.

Sociedad de Beneficencia de Lima Metropolitana. (septiembre, 2011). Plan estratégico institucional. Sociedad de Beneficencia de Lima Metropolitana. Periodo 2011-2015. Lima: autor.

Sociedad de Beneficencia de Lima Metropolitana. (2012). Memoria institucional 2012. Lima: autor.

Sociedad de Beneficencia de Lima Metropolitana. (2012). Museo Cementerio Presbítero Matías Maestro. Recuperado de http://www.sblm. gob.pe/index.php?option $=$ com_content $\& v i$ $\mathrm{ew}=$ article $\& \mathrm{id}=73 \&$ Itemid $=125$ 